Case Report

\title{
Delayed Replantation of an Avulsed Permanent Incisor Tooth
}

Soegeng Wahluyo, Ratri Anggraini, Welly Anggarani, Dwi Mulia Ramadhaniati, Udijanto Tedjosasongko Department of Pediatric Dentistry,

Faculty of Dental Medicine, Universitas Airlangga,

Surabaya - Indonesia

\begin{abstract}
Background: Incidence of dental avulsion is 1-11\% of all dental injuries to the permanent dentition, with the maxillary central incisor being the most frequently involved tooth. The age group of 7-10 years appears to be most affected. If the avulsed teeth are not treated immediately, they can have a significant negative impact on the child such as functional, aesthetic and psychological disorder. Purpose: The aims of this case reports was to discuss the important role of storage medium that can influence the success of replantation treatments of avulsion teeth. Case Management: The first phase of treatment was an extra oral root canal treatment and were repositioned into the socket. Clinical and radiographic control was carried out periodically for 2 months. Discussion: Delayed replantation has a poor long-term prognosis. The periodontal ligament will be necrotic and not expected to heal. The goal in delayed replantation is to restore the tooth to the dentition for esthetic, functional, and psychological reasons and to maintain alveolar contour. Conclusion: This case reports showed the important role of storage medium that can influence the success of replantation treatments of avulsion teeth.
\end{abstract}

Keywords: tooth avulsion, delayed replantation, root canal treatment

Correspondence: Soegeng Wahluyo, Department of Pediatric Dentistry, Faculty of Dental Medicine, Universitas Airlangga, Jl. Prof. Dr. Moestopo 47 Surabaya 60132 - Indonesia, Phone: +62-31-5030255. Email: soegeng-w@fkg.unair.ac.id

\section{INTRODUCTION}

Avulsion is defined as the complete displacement of tooth out of its socket ${ }^{1,2}$. These cases are unique because the avulsed teeth were carried with different storage medium but were treated in same methods. The first case, the tooth was carried in dry conditions (unhygienic) and the second case were soaked in natrium chloride. The prognosis for success of replantation attachment is greatly dependent on the extraoral dry time to which the avulsed tooth is exposed and placement in a storage medium that is capable of maintaining the viability of the PDL cells ${ }^{3,4}$. The prognosis of these two case are interesting to follow. The aims of these case reports were to discuss the important role of storage medium that can influence the success of replantation treatments of avulsion teeth.

\section{CASES}

Case 1

A 9-year-old girl was referred to the Pediatric Dentistry Department, Faculty of Dental Medicine, Universitas Airlangga with a major complaint that the maxillary permanent central incisor was avulsed from the socket. The trauma occurred 24 hours ago. The tooth was then carried by her parents in plastic wrapping in dry conditions.

The intraoral examination revealed that the maxillary right permanent central incisor (tooth 11) was avulsed (Figure 1A). Periapical radiographic showed no alveolar bone wall fracture or other hard tissue injuries, the root of avulsed tooth had a closed apex.

Root canal therapy was completed extra orally. The root canals were filled with guttapercha and epoxy-amine resin (AH plus ${ }^{\circledR}$, DENTSPLY). The tooth was replanted slowly in position inside the socket, under local anesthesia using epinephrine. The tooth was 


\section{Indonesian Journal of Dental Medicine}

Volume 1 Issue 1 2018; 10-13

stabilized using an orthodontic bracket and 0,014 inch NiTi arch wire technique from teeth 13 to 23 .

Patient was instructed to promote oral hygiene and soft diet, then prescribed with Albiotin at a dose of $300 \mathrm{mg} /$ day, Cataflam at a dose $75 \mathrm{mg} /$ day and Paracetamol at a dose $750 \mathrm{mg} /$ day. The patient was reviewed after 1 day, 1 month and 2 months (Figure 1B-1D).
Patient were cooperative and obey the oral hygiene instructions. The avulsed tooth remains in a stable functional position and radiographic examination showed apical healing of periodontal ligaments around the tooth begin to form (Figure 2A-2D). The splinting will be removed after a period of 3 months.
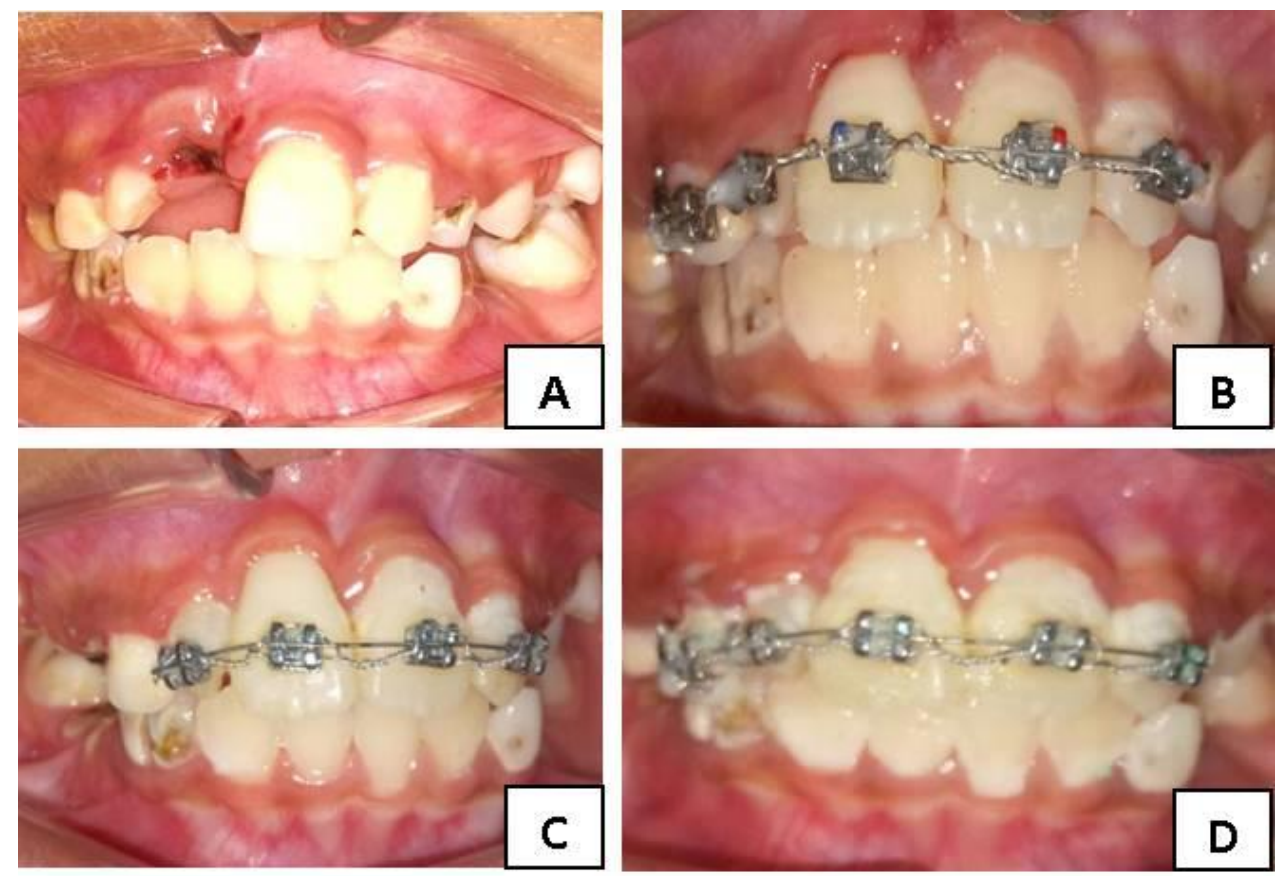

Figure 1. Frontal view, A. Photograph before replantation of avulsed tooth; B. one day after replantation; C. one month after replantation, D. two months after replantation
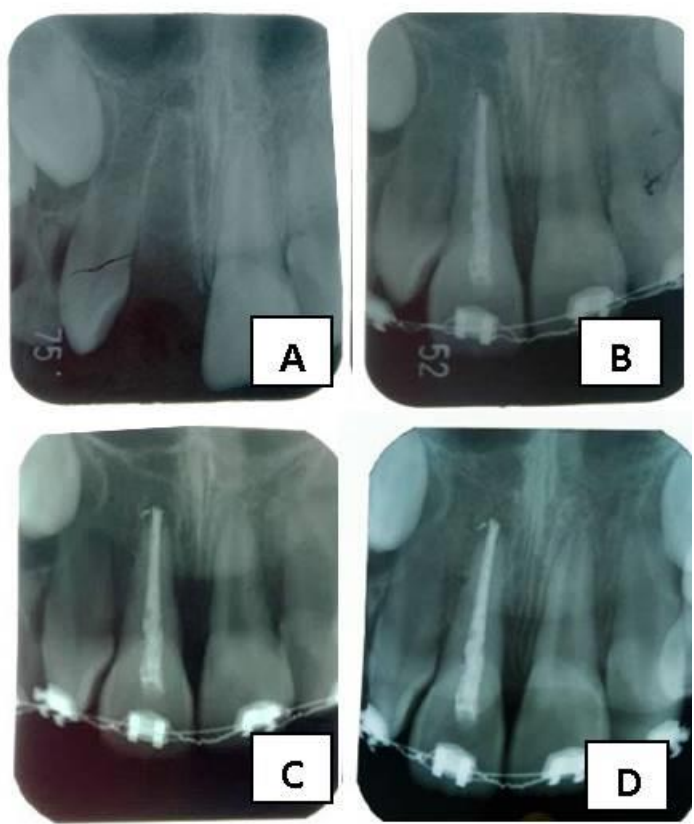

Figure 2. Periapical radiograph, A. Periapical radiograph before replantation of avulsed tooth; B. 1 week; C. 1 month D. 2 months after replantation of avulsed tooth. 
Case 2

A 10-year-old boy came to the Department of Pediatric Dentistry, Faculty of Dental Medicine, Universitas Airlangga, with chief complain of two permanent maxillary central incisors detached from the socket due to falling on the school yard. The tooth was soaked in Natrium Chloride as a containing media transport for 24 hours outside the mouth. The intra oral examination showed that there was a fracture in one third of the clinical crown teeth 11 and no fracture of clinical crown and root of teeth 21 (Figure 3A).
Root canal therapy was completed extra orally by taking all the precautions to protect the viability of the periodontal ligament. The root canals were filled with gutta-percha and epoxy-amine resin (AH plus®, DENTSPLY). Glass ionomer cement was used to restore the access cavity temporarily. The tooth was replanted slowly in position inside the socket, with slight digital pressure under local anesthesia. The tooth was stabilized using an orthodontic bracket and 0,014 inch NiTi arch wire technique from teeth 14 to 24 (exclude primary canines) (Figure 3B). The patient was reviewed after 1 day, 1 week, 1 month (Figure 3C-3D).
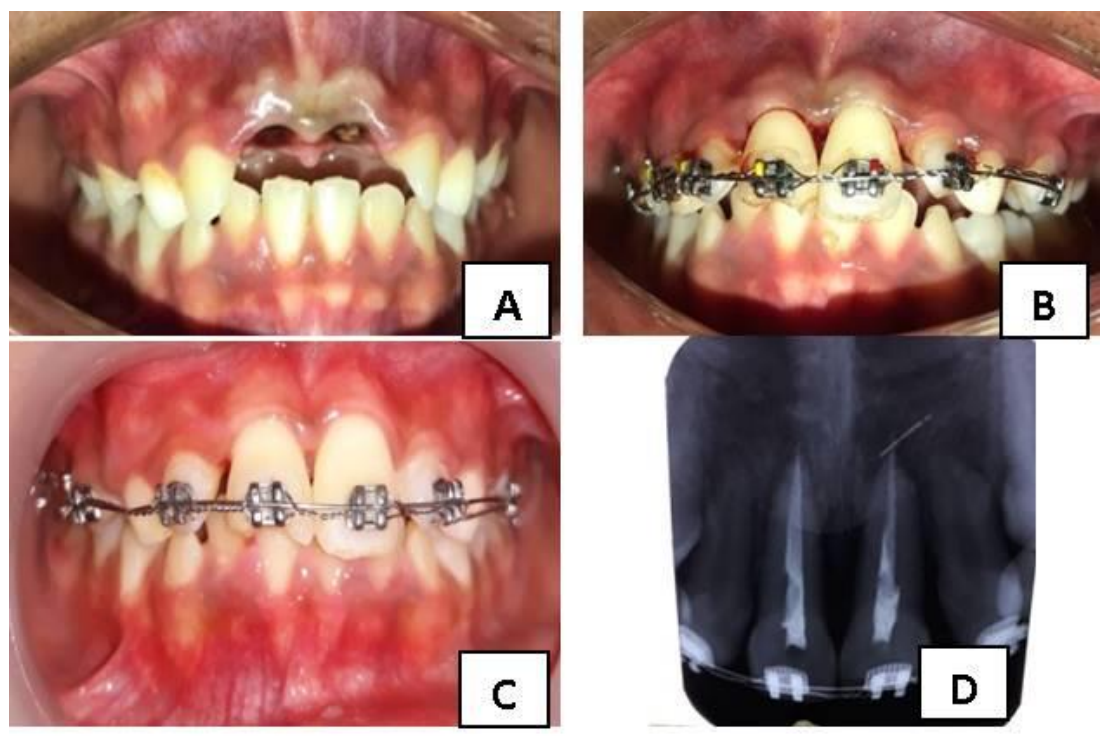

Figure 3. A. Photograph before replantation of avulsed tooth; B. Splinting of the avulsed tooth with orthodontic bracket and 0,014 inch NiTi arch wire from 14-24; C. Photograph after 1-month replantation; D. Periapical radiograph 1 month after replantation of avulsed

\section{DISCUSSION}

Tooth avulsion is the most serious of all dental injuries. The maxillary central incisors are the most prone teeth for avulsion, due to traumatic sports and injuries. The prognosis of replantation of tooth depends on the treatment of the avulsed tooth before replantation, the extra-alveolar time, the storage medium, and the patient's general health. The ideal treatment for an avulsed tooth is immediate replantation. However, it cannot always be carried out immediately. Keeping the avulsed tooth in appropriate transport media have been correlated with successful outcomes. The extra-oral period significantly affects the outcome and has a direct correlation with the survival of PDL cells ${ }^{5,6}$.

It is important for the clinician to roughly assess the condition of the cells by classifying the avulsed tooth into one of the following three groups before starting treatment: the PDL cells are most likely viable (i.e., the tooth has been replanted immediately or after a very short time at the place of accident); the PDL cells may be viable but compromised (the tooth has been kept in storage medium e.g., HBSS, saline, milk, or saliva and the total dry time has been $<60 \mathrm{~min}$ ); the PDL cells are non-viable (when the total extra-oral dry time has been >60 min regardless of if the tooth was stored in an additional medium or not) ${ }^{7}$. 
Delayed replantation has a poor long-term prognosis. The periodontal ligament will be necrotic and not expected to heal. The goal in delayed replantation is to restore the tooth to the dentition for esthetic, functional, and psychological reasons and to maintain alveolar contour. The eventual outcome will be ankylosing and resorption of the root $^{7}$.

If the tooth has been dry for more than $60 \mathrm{~min}$ before replantation, the root canal treatment may be done extra-orally prior to replantation or later. Because there were no chances of obtaining pulp space revascularization and the periodontal ligament will be necrotic and not expected to heal. In this case the avulsed tooth had a closed apex. Replanted immature teeth had less than 50\% survival after 10 years. Replanted incisors with open apices had a significantly reduced survival, and the relative risk of failure was 4.2 times greater in immature than in mature incisor ${ }^{8}$. In these two cases, since the extra oral time was more than 24 hours, it was possible that the tooth had lost its vitality, hence root canal treatment was completed before replantation of tooth in the socket.

For two cases above, endodontic treatment has been done using gutta-percha as root canal filling materials and epoxy-amine resin (AH plus®, DENTSPLY) as the sealer. Guttapercha was chosen because its strength: low tissue toxicity, good adaptation to the root canal walls, heat or chemical softening and plasticization, good radio-opacity, physicochemical stability, and ease of removal if needed. Epoxy-amine resin sealer have been used because of their reduced solubility, better apical seal and micro retention to root canal dentin. Due to its excellent properties, such as low solubility, small expansion, adhesion to dentin and very good sealing ability, AH Plus is looked as a "gold Standard" sealer ${ }^{9,10}$. This case reports showed the important role of storage medium that can influence the success of replantation treatments of avulsion teeth.

\section{REFERENCES}

1. Holan G, McTigue D. Introduction to dental trauma: Managing traumatic injuries in the primary dentition. Pediatric Dentistry: Infancy through Adolescence 4th ed 2005, Elsevier Saunders St.Louis: p. 236-56.

2. McIntyre J, Lee J, Trope M, Vann WJ. Permanent tooth replantation following avulsion: Using a decision tree to achieve the best outcome. Pediatr Dent 2009;31(2):137-44

3. Bazmi, BA., Singh, AK., Kar, S., Mubtasum, H. Storage media for avulsed tooth : A review. Indian Journal of Multidiciplinary Dentistry 2013;3:741-74

4. Malhotra N. Current developments in interim transport (storage) media in dentistry: an update. Brit Dent J 2011;211:29-33.

5. Majd NM, Zohrehei H, Darvish A, Homayouni H, Adell M. Continued Root Formation after Delayed Replantation of an Avulsed Immature Permanent Tooth. Case Reports in Dentistry. Hindawi Publishing Corporation. Vol. 2014, Article ID 832637, 5 pages

6. Savas S, Kucukyilmaz E, Akcay M, Koseoglu S. Delayed Replantation of Avulsed Teeth: Two Case Reports. Case Reports in Dentistry Vol. 2015, Article ID 197202, 5 pages

7. Andersson L et al. International Association of Dental Traumatology Guidelines for The Management of Traumatic Dental Injuries: Avulsion of Permanent Teeth. Dental Traumatology 2012;28: 88-96; doi: 10.1111/j.1600-9657.2012.01125.x

8. Kostka E, Meissner S, Finke $\mathrm{CH}$, Mandirola M, Preissner S. Multidisciplinary Treatment Options of Tooth Avulsion Considering Different Therapy Concepts. The Open Dentistry Journal 2014;8:180-183

9. Bhavana V, DurgaRao PV, Kumari A, Leneena G. Delayed replantation of an avulsed tooth-A case report. IP Annals of Prosthodontics \& Restorative Dentistry 2017;3(4):127-129

10. Panzarini SR, Trevisan CL, Brandini DA, Poi WR, Sonoda CK, Luvizuto ER, Vendrame dos Santos CL. Intracanal dressing and root canal filling materials in tooth replantation: a literature review. Dental Traumatology 2012;28:42-48; doi: 10.1111/j.1600-9657.2011.01023.x 